\title{
Wind Tunnel Test of the Icing Characteristics of Airfoil Rotating around a Vertical Axis
}

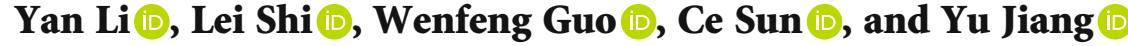 \\ College of Engineering, Northeast Agricultural University, Harbin 150030, China \\ Correspondence should be addressed to Yan Li; liyanneau@163.com
}

Received 6 April 2020; Revised 13 September 2020; Accepted 16 October 2020; Published 3 November 2020

Academic Editor: Ashwani K. Gupta

Copyright ( 2020 Yan Li et al. This is an open access article distributed under the Creative Commons Attribution License, which permits unrestricted use, distribution, and reproduction in any medium, provided the original work is properly cited.

\begin{abstract}
To determine the process of icing on the rotating machinery, an icing experiment on a rotating airfoil blade was carried out in this paper. First, an icing wind tunnel was fabricated, and its conditional parameters were calibrated. The calibration results showed that the performance of this icing wind tunnel was reliable and stable. The experimental temperature was $-15^{\circ} \mathrm{C}$, and the $\mathrm{MVD}$ was $50 \mu \mathrm{m}$. Then, an icing experiment on the rotating blade with the NACA0018 airfoil was carried out. The characteristics of icing, including icing distribution, growth rate of icing, and thickness of the ice layer, were defined and quantitatively analyzed under different tip speed ratios and setting angles. The results show that the type of icing changes from rime ice to glaze ice with an increase in the tip speed ratio. The dimensionless icing area and dimensionless thickness of the ice layer both increase with an increase in the icing time. The growth rate of icing increases rapidly at the initial icing stage and then decreases dramatically under each tip speed ratio condition.
\end{abstract}

\section{Introduction}

Icing on some machinery with airfoil structures, such as wind turbines, fixed-wing airplanes, and helicopter rotors, will result in reductions in performance and safety. Wind energy is a kind of renewable energy that is widely used all over the world $[1,2]$. However, when a wind turbine is located in a high-latitude region with low temperatures and high humidity, icing occurs on the blade surface and changes its aerodynamic profile, mass distribution, and surface roughness, which shortens the life span of the blade and degrades the power generation efficiency of the wind turbine $[3,4]$. Similarly, when fixed-wing airplanes and helicopters operate at high altitudes and encounter icing events, the safety of the aircraft is threatened by icing $[5,6]$. Therefore, research into the process and shape of the icing on these machines provides the foundation for anti- and deicing methods.

Some scholars have researched the process of icing on these machines. Yan et al. examined the ice accretion on the blade surface of NACA7715 airfoil in a self-developed icing wind tunnel. The icing rates and icing areas on the blade elements, which were fixed under several angles of attack, were measured [7]. Xian modeled and simulated the icing procedures of supercooled water droplets impacting on the NACA 0012 and NLR7301, including the trajectory equation and heat transfer model [8]. There are two main research methods: one is carried out in an icing wind tunnel, and the other involves numerical simulation. Taylor proposed the experimental research method of icing on aircraft by an icing wind tunnel as early as 1940 [9]. Long et al. optimized the heat transfer of an anti-icing component for a helicopter rotor by a mathematical model and validated the theoretical results by experimentation. The experiment was carried out in a refrigerator system, which is different from an icing wind tunnel system [10]. Ruff conducted research on the similarity of icing on airfoils with different scales and derived icing scaling equations. The theoretical findings were validated in an icing wind tunnel [11]. Wang simulated the icing shape of a horizontal axis wind turbine blade by quasi-three-dimensional numerical simulation. The simulation results were compared with experimental results, which were acquired by an icing wind tunnel [12]. Flemming et al. carried out icing tests on a rotor of a S92A helicopter in the static states for different temperatures, wind velocities, liquid 


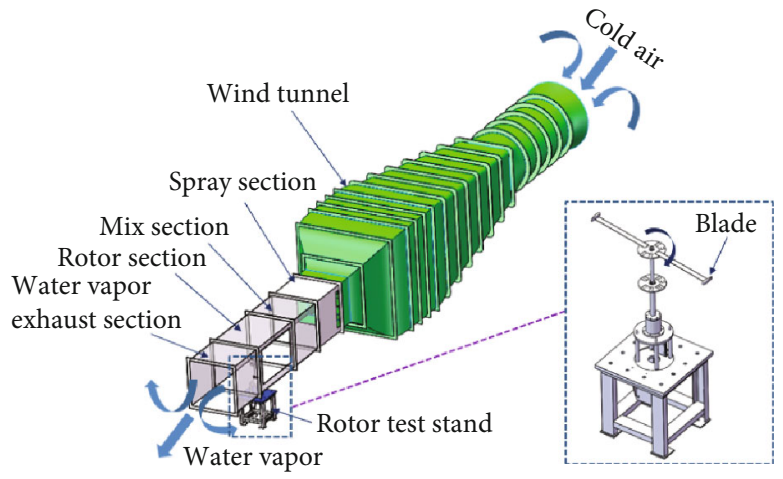

(a) Icing wind tunnel

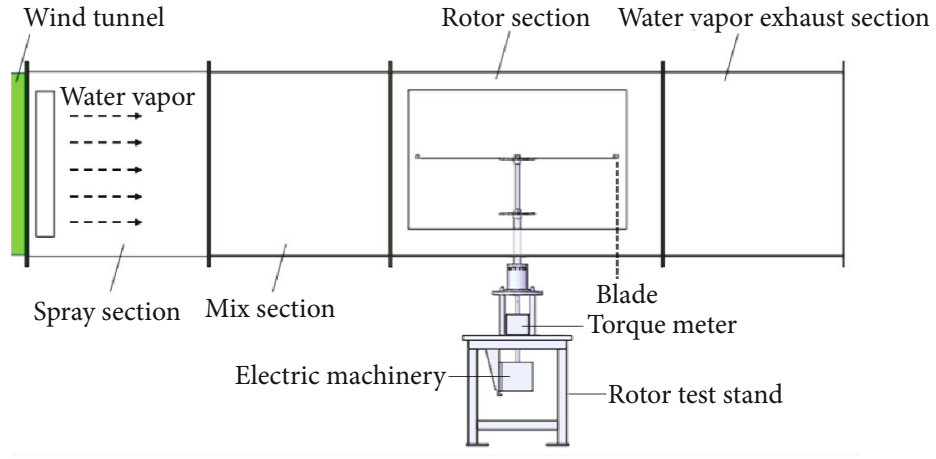

(b) Experimental device

FIgURE 1: Icing wind tunnel system facility.

TABLE 1: Key characteristic parameters of the icing wind tunnel.

\begin{tabular}{lc}
\hline Items & Values \\
\hline Outlet size of the icing wind tunnel & $1 \times 1 \mathrm{~m}^{2}$ \\
Wind speed & $0 \sim 20 \mathrm{~m} / \mathrm{s}$ \\
Dynamic stability & $\eta \leq 1 \%$ \\
Pressure field coefficient & $\mu_{I} \leq 1 \%$ \\
\hline
\end{tabular}

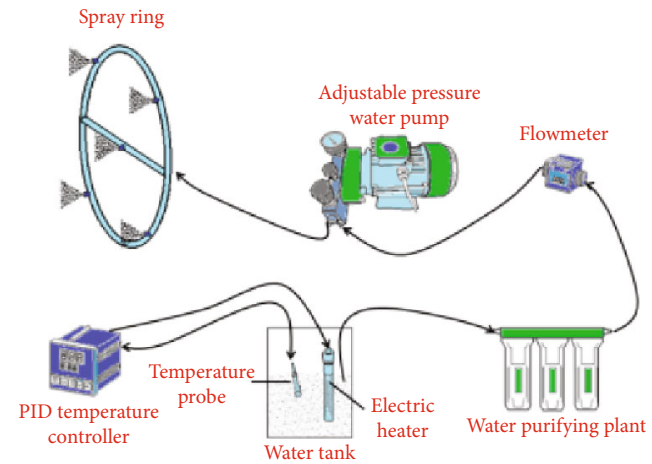

FIGURE 2: Spray system.

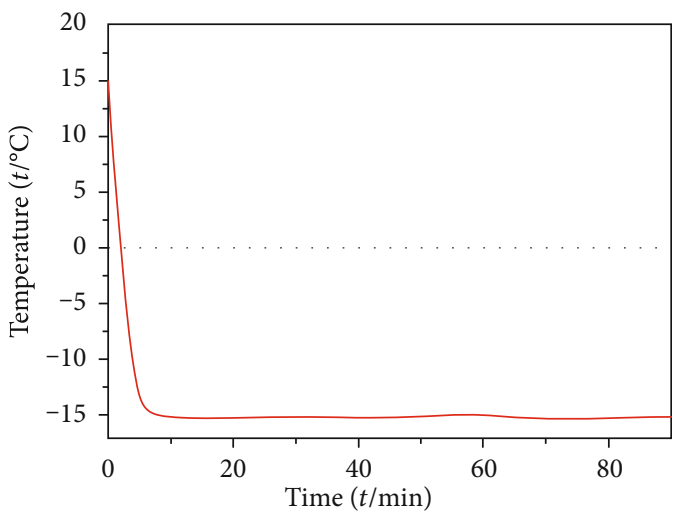

Figure 3: Variation of temperature in 90 minutes. water contents (LWCs), and medium volume droplets (MVDs) [13]. Waldman and $\mathrm{Hu}$ and Yang et al. examined the dynamic process of ice accretion and the degradation of the aerodynamic performance of a UAS propeller model by a high-speed camera and particle image velocimetry (PIV) $[14,15]$. Han et al. conducted scaled ice accretion experiments on a rotating wind tunnel blade, which was from an NREL Phase VI rotor and compared the experimental data with simulated data by LEWICE [16]. Kraj and Bibeau carried out experiments on an anti-icing method by using an icing wind tunnel, including a thermal deicing method, a surface coating method, and a thermface deicing method [17]. Yan et al. researched the ice accretion on the blade surface of a straight-bladed vertical axis wind turbine rotating at a low tip speed ratio by using a self-developed icing wind tunnel. The results, including icing shape, icing area, and icing ratio, were different from the results obtained in a nonrotating state [18]. These research findings provide the theoretical and experimental foundations for anti- and deicing technology. However, the airfoil blades in these research studies were in the nonrotating state or at a low tip speed ratio. The icing process and characteristics of the airfoil blade at a high tip speed ratio are seldom investigated by experimentation.

In this paper, a self-developed icing wind tunnel was designed and fabricated, based on a conventional wind tunnel and a low natural temperature. The parameters of this experimental system were calibrated in previous work [19]. Using the icing wind tunnel, icing tests were conducted on a blade with NACA 0018 airfoil rotating around a vertical axis at high tip speed ratios and different setting angles. The results provide experimental foundations for researching anti- and deicing methods.

\section{Experimental System}

2.1. Building of an Icing Wind Tunnel. To investigate the process of icing on a blade surface, an icing wind tunnel experimental system based on a conventional wind tunnel was designed and built, and the computer model is shown in Figure 1.

Figure 1 shows that the icing wind tunnel system is composed of a wind tunnel, a spray system, a mix section, a rotor section with a rotor test stand, a water vapor exhaust section, 


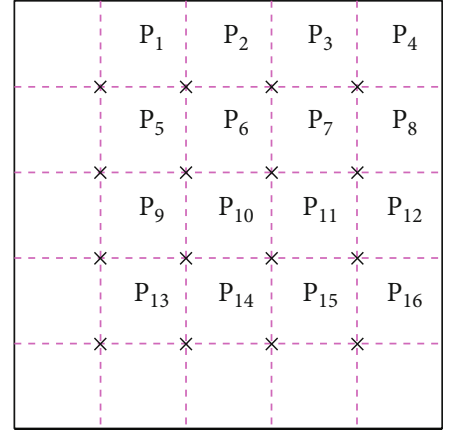

FIGURE 4: Measuring points at a cross-section for temperature calibration.

and an image capture system. The cold air in winter from the outside environment is pulled into the wind tunnel as the source of low temperatures. This procedure greatly simplifies the components of an icing wind tunnel system and eliminates the cooling system with its associated high cost. The key characteristic parameters of the icing wind tunnel are listed in Table 1.

The spray system, used to generate water droplets, is composed of a spray ring, a water pump, a flowmeter, a water purifier, a water tank, and a thermometer, as shown in Figure 2. The spray ring has five nozzles, and the diameter of the orifice in each nozzle is $0.3 \mathrm{~mm}$. The water pump, which can modulate the water pressure, pulls water from the water bank to the nozzle and sprays it out at a certain pressure. The modulation range of pressure is 0-7 MPa. For safety and reliability of the spray system, the water pressure of the spray system in this icing test is $2 \mathrm{MPa}$. When the water pressure is $2 \mathrm{MPa}$, the medium volume diameter (MVD) of the water droplet is about $50 \mu \mathrm{m}$. The calibration method and process are elaborated in Section 2.2.4. The flowmeter is used to control the rate of flow to modulate the liquid water content (LWC). The water tank is used to store water, which is mixed with ice during the experiment to keep the water being near to $0^{\circ} \mathrm{C}$.

In this subsystem, the nozzle array is installed at the outlet of a conventional wind tunnel. The nozzle array injects microsized water droplets into the airflow. The size of the water droplets and the LWC are adjusted by modulating the pressure of the water pump. When the cold air is brought into the wind tunnel from the outside environment, the temperature in the air channel begins to decrease. As the temperature stabilizes, the spray system begins working, and the water droplets are injected and mixed with the cold air. In this process, the water droplets release heat and become supercooled water droplets. When the supercooled water droplets impact the surface of the research object, icing is initiated on the object surface. The rest of the supercooled droplets are exhausted to the outside environment with the airflow.

2.2. Calibration of the Icing Wind Tunnel System. Before the experiment, four key system parameters of the icing wind tunnel are calibrated based on previous work. In this paper, the system parameters that are adjusted include the experi- mental temperature, the flow field parameters, the liquid water content, and the medium volume droplet [19].

2.2.1. Calibration of Temperature. The test section is the key component of the icing wind tunnel system. Therefore, the stability and distribution of temperature in the test section must be calibrated. In this paper, a temperature sensor, whose model number is HTC-1, is selected to measure the temperature stability and distribution. First, the temperature at the center point is measured at 90 minutes, and the variation of the temperature is shown in Figure 3. The result shows that the temperature rapidly decreases from room temperature to $-15^{\circ} \mathrm{C}$ in approximately 18 minutes and remains stable throughout the entire period.

Due to the working characteristics of axial flow fans, the effective area of the conventional wind tunnel is approximately $80 \%$. Therefore, the distribution of temperature at the cross-section near the rotor test stand must be calibrated. In this paper, 16 measuring points are selected at the crosssection, as shown in Figure 4. The temperatures of these 16 points are measured, and a cloud picture of the temperature is plotted by MATLAB, as shown in Figure 5.

As shown in Figure 5, the temperature gradually increases from the center to the wall of the wind tunnel. The distribution of temperatures in the central area is more uniform than the distribution in the rest of the areas. The uniform area accounts for approximately $75 \%$ of the total cross-section area. Therefore, the icing test should be carried out in this region. The rotating radius of the blade in the icing test should be shorter than $750 \mathrm{~mm}$. These results show that the temperature is stable and distributed uniformly in the test region. Thus, the icing wind tunnel satisfies the experimental temperature needed for the icing test.

2.2.2. Calibration of the Flow Field Parameters. The stability and distribution of the flow field in the test section are important to the experimental results. Therefore, calibration of the flow velocity in the test section should be performed. In this paper, the flow velocity is measured by a hot wire wind speed sensor, whose model is testo405i, made by the Testo Company. First, the flow velocity at the center point of the cross-section is measured for 90 minutes, and the variation in the flow velocity is shown in Figure 6.

As shown in Figure 6, the flow velocity rises in a short time from 0 to $4 \mathrm{~m} / \mathrm{s}$ and remains stable thereafter. To obtain the distribution of the flow field in the test section, the flow velocities at 16 points, which are the same as the points used in the temperature calibration, are measured. Then, a cloud picture of the flow velocity is plotted by MATLAB, as shown in Figure 7.

As shown in Figure 7, the flow velocity in the central area of the test section is relatively uniform and higher than that in other areas. The uniform area in the center is approximately $70 \%$ of the total cross-sectional area. Therefore, the icing test should be carried out in this region. These results show that flow velocity in the test section of the icing wind tunnel is stable and distributed uniformly in the test section. The flow velocity in the icing wind tunnel satisfies the demands of the icing test. 


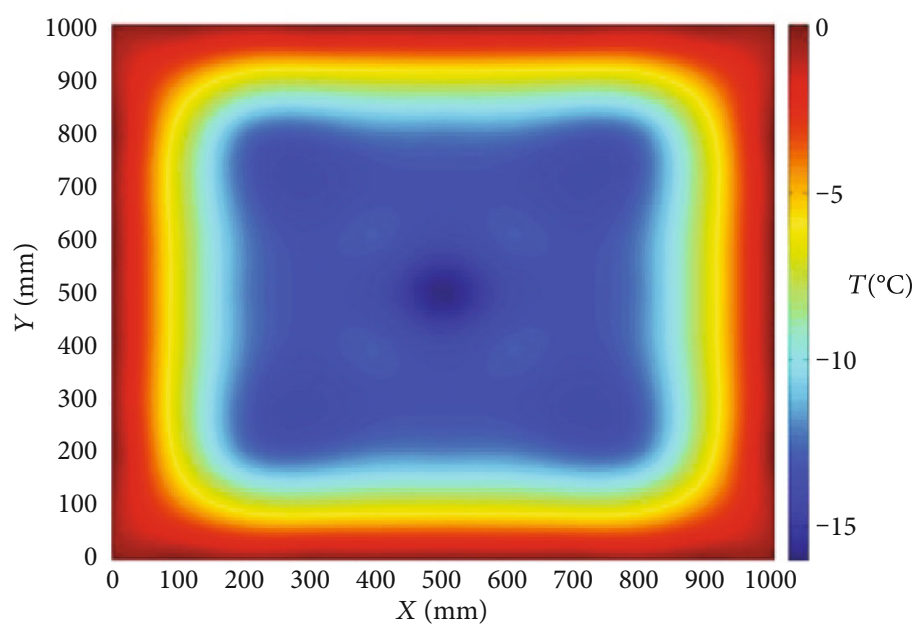

FIgURE 5: Cloud picture of temperature at a cross-section.

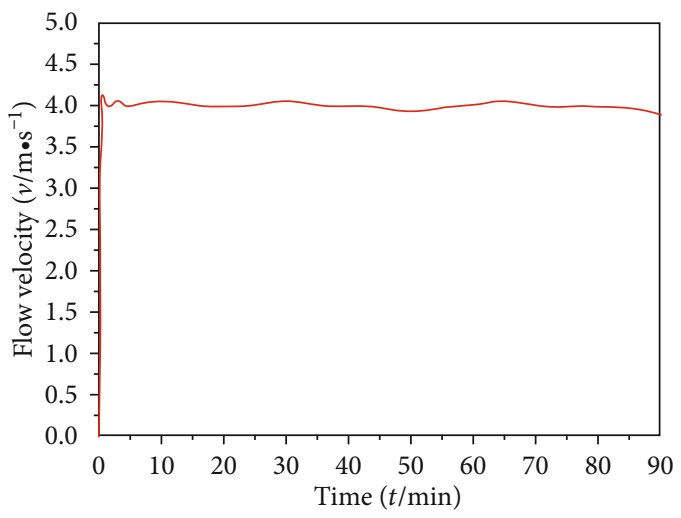

FIgURE 6: Variation of flow velocity in 90 minutes.

2.2.3. Calibration of Liquid Water Content. Liquid water content (LWC) is a key experimental parameter in icing tests, which influences the shape of the icing on the model surface. In this paper, the LWC is acquired by the grid method, which measures the thickness of the icing on the grid, as shown in Figure 8. In this paper, the grid is made of Plexiglas.

The cloud picture of the LWC at the cross-section in the test section is shown in Figure 9.

As shown in Figure 9, the LWC is also distributed uniformly in the central area of the cross-section. The uniform area is approximately $75 \%$ of the total cross-section. Therefore, the icing test should be carried out in this region.

2.2.4. Calibration of the Medium Volume Droplet. The medium volume droplet (MVD), also a key parameter in the icing test, influences the shape of the icing. In this paper, by comparing the coefficient of local water droplet collection $\beta$ between the icing test and the simulation, the MVD in the icing test can be calculated and acquired indirectly from the simulation [20]. The calibration samples selected in this paper are two cylinders, as shown in Figure 10, and both are composed of Plexiglas.

As shown in Figure 10, the diameters of the two cylindrical samples are both $30 \mathrm{~mm}$, and their heights are both
$150 \mathrm{~mm}$. The simulation with the same conditions used in the icing test is carried out, and the meshing result is shown in Figure 11.

By simulation, the coefficient of local water droplet collection under different MVDs, which are $30 \mu \mathrm{m}, 40 \mu \mathrm{m}$, $50 \mu \mathrm{m}, 60 \mu \mathrm{m}$, and $70 \mu \mathrm{m}$, is calculated. The coefficients of the local water droplet collection of simulations and the experiment are shown in Figure 12(a). It is the plot of the coefficient of local water droplet collection on any portion of the cylinder. The coefficient of local water droplet collection represents the rate of accumulation per unit area per second at any point on the surface. The ordinate is the value of the coefficient of local water droplet collection, and the abscissa is the position along the diameter of the cylinder, which is shown in Figure 12(b).

As shown in Figure 12(b), the cylinder surface is divided into two regions by horizontal centerline. They are upper surface and lower surface, respectively. In Figure 12(a), the abscissa represents the position or location along the diameter of the cylinder which is vertical to flow direction. The positive value of abscissa indicates the positive direction as shown in Figure 12(b), and the negative one indicates the negative direction. The origin is the location of zero point, which is the center of the cylinder.

In Figure 12(a), the MVD is the abbreviation of medium volume diameter, which is from $30 \mu \mathrm{m}$ to $70 \mu \mathrm{m}$. The range of it is selected according to previous work. It indicates that the coefficient of local water droplet collection $\beta_{0}$ in each MVD condition is calculated by the simulation method. All the other simulation conditions are the same as the ones in the calibration experiment. The coefficient of local water droplet collection in the calibration experiment $\beta$ is calculated by the following formulae $[21,22]$ :

$$
\beta=\sum_{i=1}^{N} n_{i} \beta_{i}
$$

where $\beta$ is the coefficient of local water droplet collection in the calibration experiment; $n_{i}$ is the mass ratio of water 


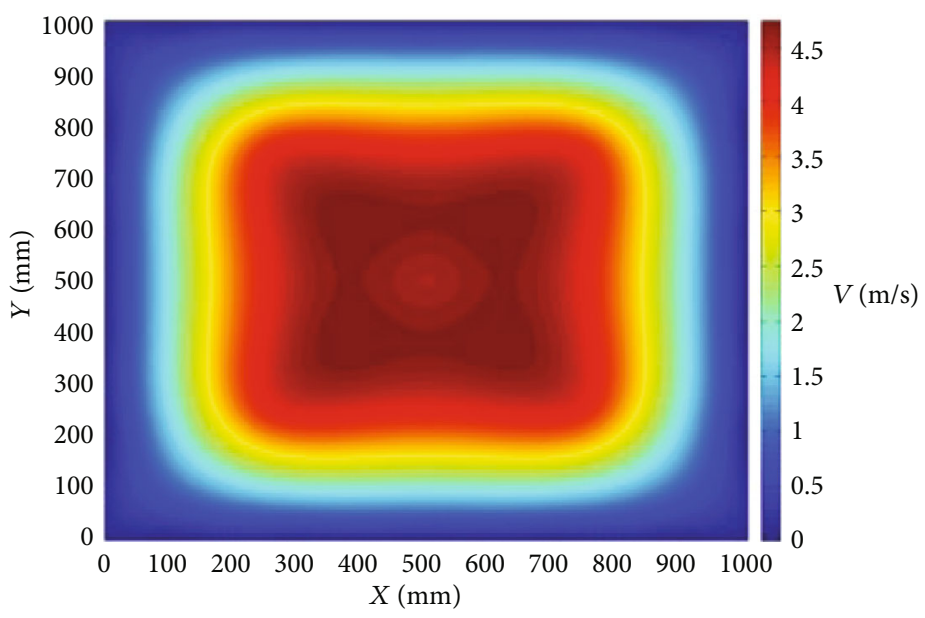

Figure 7: Cloud picture of flow velocity.

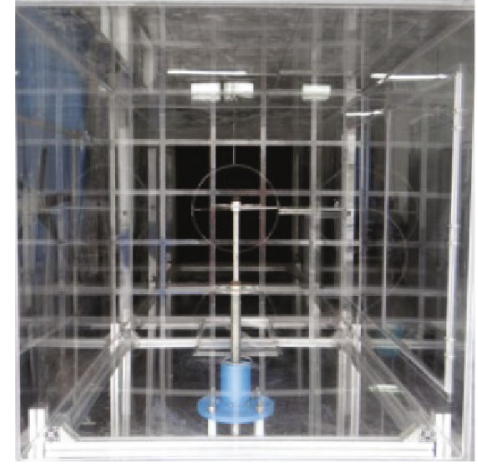

FIGURE 8: Calibration of the LWC by the grid method.

droplets; $\beta_{i}$ is the coefficient of local water droplet collection in each mass ratio.

$$
\frac{\beta_{01}}{\beta_{02}}=\frac{\beta_{1}}{\beta_{2}}=\frac{h_{1} V_{2}}{h_{2} V_{1}},
$$

where $\beta_{01}$ and $\beta_{02}$ are the coefficients of local water droplet collection of simulation under $V_{1}$ and $V_{2}$ conditions; $\beta_{1}$ and $\beta_{2}$ are the coefficients of local water droplet collection of calibration experiment under $V_{1}$ and $V_{2}$ conditions; $V_{1}$ and $V_{2}$ are wind speeds; $h_{1}$ and $h_{2}$ are thicknesses of icing layers.

After calculations, the results of simulations and experiments are plotted together as shown in Figure 12(a). By comparison of the curves between simulations and experiment, the value of MVD in the calibration experiment can be inferred.

As shown in Figure 12(a), the curve of the coefficient of local water droplet collection in the icing test locates between the ones of $40 \mu \mathrm{m}$ MVD curve and $60 \mu \mathrm{m}$ MVD curve in the simulations. Therefore, MVD is considered to be on the order of $50 \mu \mathrm{m}$ in this icing wind tunnel.

The values of the calibrated parameters mentioned above are summarized and listed in Table 2.

\section{Icing Experiment}

3.1. Experimental Equipment. A sketch map of the rotor test stand and the icing shape collection system is shown in Figure 13. As shown in Figure 13, the rotor test stand is installed in the test section, and the rotating radius of the blade sample is $350 \mathrm{~mm}$. Two of the same blades made of aluminum with NACA0018 airfoil are symmetrically fixed at the end of cantilever beams. The chord length of blade $\mathrm{C}$ is $100 \mathrm{~mm}$, and the thickness $H$ is $10 \mathrm{~mm}$. The icing shape collection system comprises a high-speed camera and a computer. The high-speed camera, model Phantom 5.1 made by Vision Research, Inc., is used to capture the image of the shape of the icing and sends the electronic image to the computer.

3.2. Experimental Scheme. The tip speed ratio is a key parameter in the field of wind energy. In previous research, the law of ice accretion on the blade surface under a low tip speed ratio was analyzed. In this paper, the characteristic of ice accretion under the condition of a high tip speed ratio is tested and analyzed. The formula for the tip speed ratio is expressed as follows:

$$
\lambda=\frac{U}{v}
$$

where $U$ is the tangential velocity of the blade and $v$ is the wind velocity.

In this paper, five tip speed ratios and two setting angles are selected. The moving trajectory of the blades and the area enclosed by the trajectory, which is divided into two regions by a boundary between $0^{\circ}$ and $180^{\circ}$ azimuth angles, are shown in Figure 14. The blue area is called the backward motion area, and the orange area is called the forward motion area. In the backward motion area, the flow direction is the same as the direction of the linear velocity component of the blade along the boundary. In the forward motion area, the flow direction is opposite to the direction of the blade linear velocity component along the boundary. The setting angle $\alpha$ is the included angle between the chord line of the blade and the horizontal 


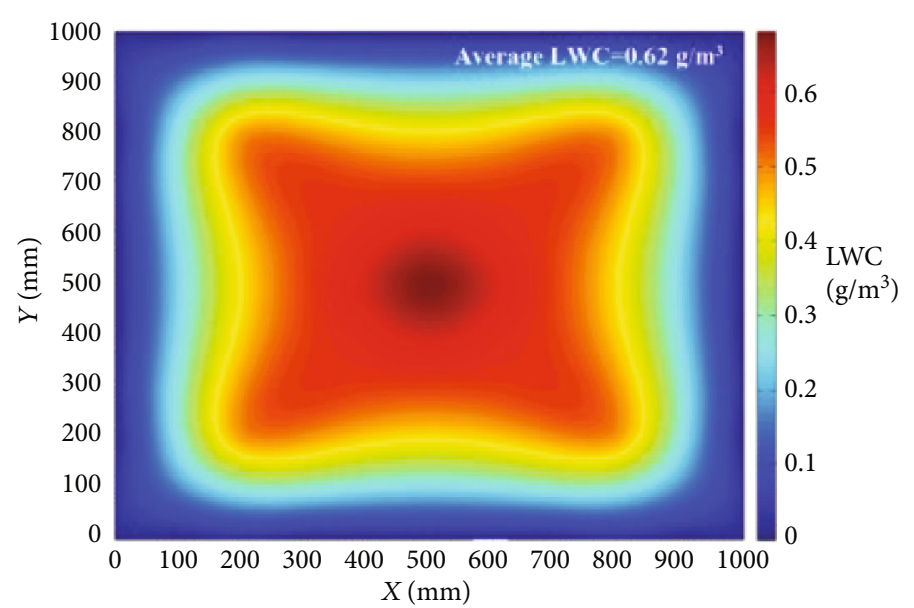

FIgURE 9: Cloud picture of the LWC at the cross-section in the test section.

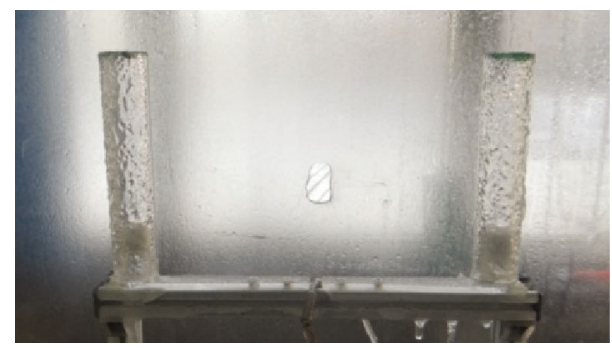

Figure 10: Cylinder sample for calibration.

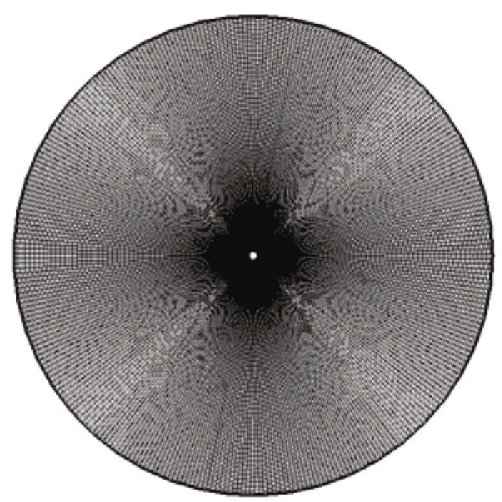

FIgURE 11: Meshing result.

plane as shown in Figure 14. For exploring the influence of the icing time on the law of ice accretion, different icing time lengths are selected under different tip speed ratios. The experimental scheme is shown in Table 3.

Before each icing test, the blade was cooled by cold air without water droplets in order to make the temperature of the blade equal to the ambient temperature in the test section. After five minutes, the spraying system was initiated to carry out the icing test.

\section{Experimental Results}

4.1. Type of Ice. In the experiment, there are three types of ice present on the blade surface at different setting angles and tip speed ratios. They are rime ice, glaze ice, and mixing ice, which are shown in Figure 15. As shown in Figure 15(a), rime ice is a dry, milky, and opaque ice deposit which usually occurs at low airspeed, low temperature, and low liquid water content. It is characterized by the instantaneous freezing of the incoming supercooled water droplets as soon as they hit the surface. As shown in Figure 15(c), glaze ice is wet growth ice formed at a temperature of $0^{\circ} \mathrm{C} \sim-10^{\circ} \mathrm{C}$ and high liquid water content. It has a clear appearance and a density closer to that of cloud water indicating the wet nature of this icing type [5]. As shown in Figure 15(b), the mixing ice has both the rime ice and the glaze ice. The substrate ice layer is the rime ice, which presents in the initial icing time. The upper ice layer is the glaze ice, which generates in the mid- and late icing time.

According to the experimental results, when the setting angle is $0^{\circ}$, rime ice is present on the blade surface at the tip speed ratios of 0 and 1 , and mixing ice is present at the tip speed ratios of 3,5 , and 7 . When the setting angle is $5^{\circ}$, rime ice is also generated at low tip speed ratios, such as 0 and 1 . However, mixing ice is present at tip speed ratios of 3 and 5 , and glaze ice occurs at a tip speed ratio of 7 .

In summary, when the tip speed ratio is low, the type of icing on the blade surface is rime ice. In contrast, when the tip speed ratio is high, the types of ice are mixing ice and glaze ice. In addition, when the tip speed ratio is high (such as 3, 5, and 7), the phenomenon of ice shedding happens.

The reason for generating different types of icing is that the blade has different water droplet collection coefficients and heat transfer processes occurring under different tip speed ratios. When the tip speed ratio is low (such as 0 and 1 ), the blade captures a small number of water droplets per unit time because of the low rotational speed. As the water droplets contact the blade surface, the heat in water droplets transfers into the blade instantly, because the aluminum blade has high thermal conductivity and cold surface in rime ice condition. In this case, the water droplets freeze in a short time. The rime ice layer accretes on the blade surface, and the ice layer surface again has a low temperature. Under this condition, rime ice can be generated layer by layer. When the tip speed ratio is high (such as 3 and 5), the blade captures more 


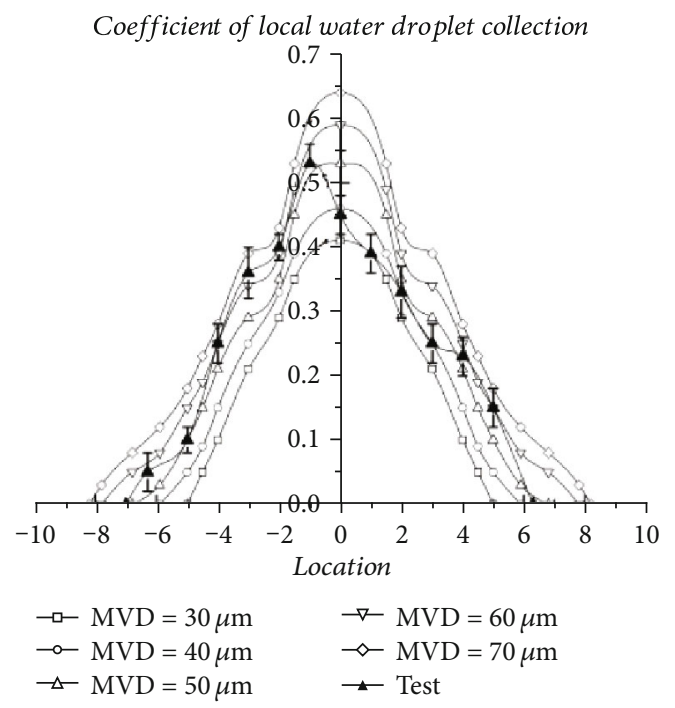

(a) Coefficients of local water droplet collection

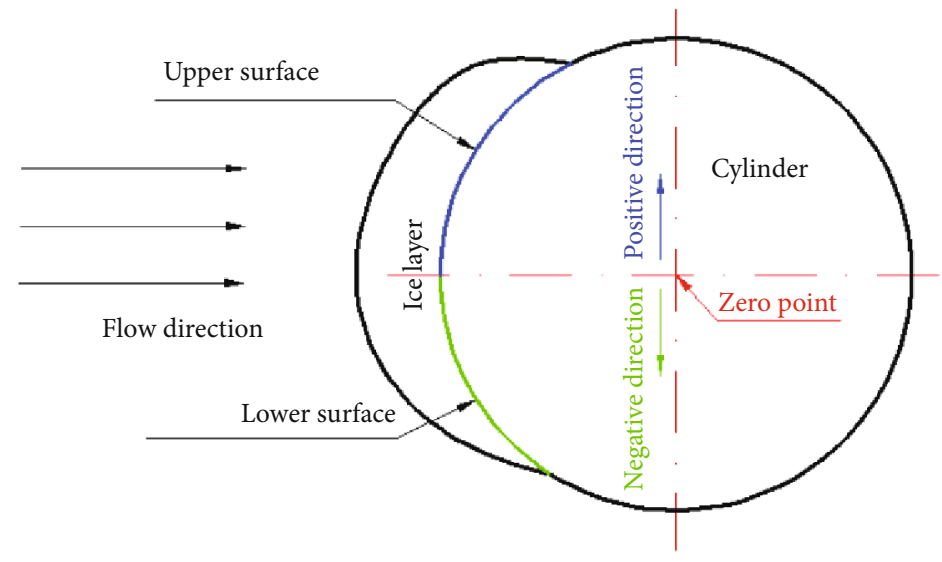

(b) Location of cylinder surface

FIGURE 12: Comparison of the coefficient of local water droplet collection between test and calculation.

TABLE 2: Values of the system parameters.

\begin{tabular}{lc}
\hline Item & Value \\
\hline Experimental temperature & $-15^{\circ} \mathrm{C}$ \\
Flow velocity & $0 \sim 15 \mathrm{~m} / \mathrm{s}$ \\
Medium volume diameter (MVD) & $50 \mu \mathrm{m}$ \\
\hline
\end{tabular}

water droplets per unit time than that in low tip speed condition. In the early icing time, the heat in water droplets hitting the aluminum blade surface can be absorbed by the blade, because of the high thermal conductivity of aluminum material. The rime ice layer is generated in this case. However, with an increase in the icing time, as the rime ice covers the whole blade surface in a short time, the subsequent large numbers of water droplets contact the rime ice layer. The rime ice layer cannot fully absorb the heat from the water droplets in a short time, because of the low thermal conductivity of ice. The temperature of water droplets is higher than the experimental temperature in the rime ice condition. Then, the glaze ice layer is generated on the rime ice layer surface. As a result, there is mixing ice presenting on the blade surface. With a continuous increase in tip speed ratio (such as 7), a larger number of water droplets can be captured by the blade per unit time. The heat in water droplets cannot be absorbed by the blade in a short time, even if the water droplets directly contact the blade surface in the early icing time. The water droplets have a higher temperature than the ambient temperature. It results in the generation of glaze ice on the blade surface during the whole icing time.

4.2. Shape of Icing. The shape of the icing of the airfoil in this paper is acquired by the CAD software. The pictures of the icing blade, at each acquisition time, were taken by a highspeed camera during the icing test. Then, the pictures with a clear profile of the icing shape were selected and imported into the CAD software. The shape of the icing is drawn by the spline curve function in CAD. After drawing, the parameters of the shape of the icing were measured by CAD. The icing shapes under different tip speed ratios and setting angles are shown in Figure 16. The ice accretion is present only on the leading edge because the linear velocity of the rotating blade is higher than the flow velocity in the backward motion area under high tip speed ratio conditions. The water droplets cannot impact on the trailing edge.

From Figure 16, the blade surface is divided into two regions: the upper surface region and the lower surface region, respectively.

As shown in Figure 16, ice accretes on the blade surface layer by layer with an increase in the icing time. When the rotational speed of the blade is 0 , the surface of the ice layer is smooth. In contrast, the surface of the ice layer is rough when the blade rotates around the vertical axis. The rotational speed has a great influence on these results.

When the setting angle is $0^{\circ}$, the areas covered by the ice layer on the upper blade surface are larger than the areas covered by the ice layer on the lower surface under different tip speed ratios because more droplets fall and impact the upper blade surface because of gravity. In addition, the areas covered by ice layers under different tip speed ratios are approximately the same. The area covered by the ice layer on the upper blade surface accounts for approximately $30 \%$ of the total upper blade surface area, and the area covered by the ice layer on the lower blade surface is approximately $15 \%$ of the total lower blade surface area. As the setting angle is $5^{\circ}$, the area covered by the ice layer on the upper blade surface is smaller than the areas covered by the ice layer on the lower surface. When the tip speed ratio is 1 , the area covered by the ice layer on the upper blade surface is larger than the areas covered by the ice layer at other tip speed ratios. Under these conditions, the icing area covering the upper blade surface is approximately $20 \%$ of the total upper blade surface area. Under other conditions, the icing area accounts for approximately $10 \%$ of the total upper blade surface area. With an 


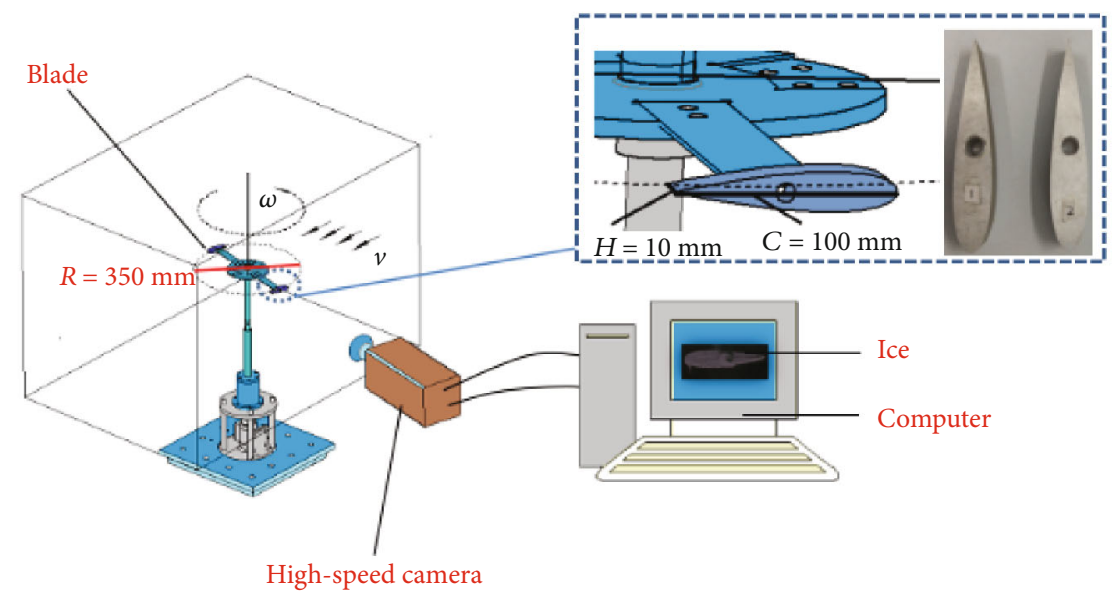

FIGURE 13: Rotor test stand and icing shape collection system.
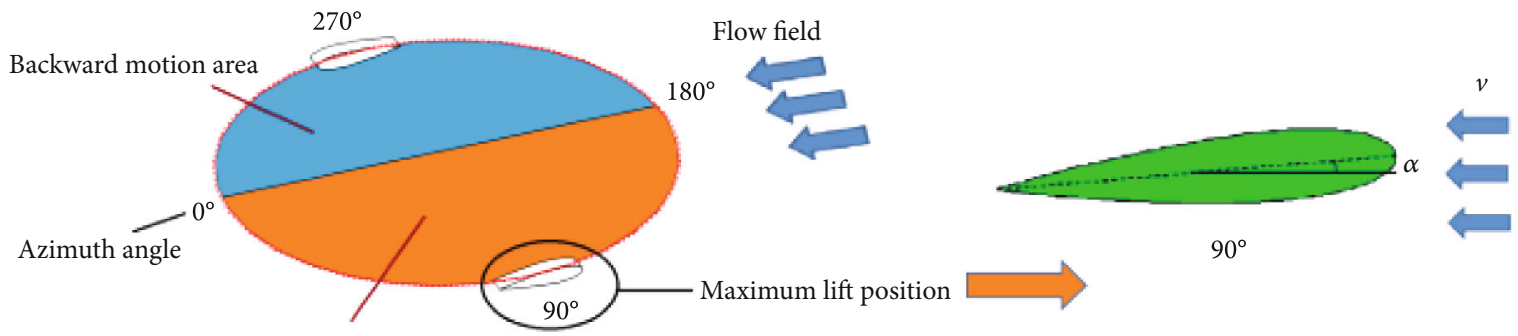

Forward motion area

FIGURE 14: Moving trajectory of blades and setting angle $\alpha$.

increase in the tip speed ratio, the icing area on the lower blade surface gradually extends to the trailing edge of the blade. When the tip speed ratio is 7 , the icing area accounts for approximately half of the total lower blade surface area.

\section{Evaluation and Analysis}

5.1. Evaluation Method. For quantitatively analyzing the characteristics of icing on the blade surface, an evaluation method was utilized based on previous work [20]. In this method, five typical parameters for evaluating icing shape were defined. Icing shape can be quantitatively analyzed and evaluated by these parameters. In this paper, two parameters (icing area and stationary point thickness) are selected to quantitatively analyze the law of icing shape on the blade surface. The definitions of these two typical parameters are shown in Figure 17.

The mathematical expression of these two dimensionless parameters is expressed as formulas (4) and (5).

$$
\begin{gathered}
\eta_{S}=\frac{S}{S_{b}}, \\
\eta_{\sigma}=\frac{\sigma}{C},
\end{gathered}
$$

where $\eta_{s}$ is the dimensionless icing area, $S$ is the crosssectional area of the icing layer, $S_{b}$ is the cross-sectional area of the blade, $\eta_{\sigma}$ is the dimensionless stationary point thick- nesses, $\sigma$ is the stationary point thickness, and $C$ is the chord length of the blade.

According to the data acquired by the icing tests, the growth rate of the icing under different conditions shows discrepancies. Quantitative analysis of the growth rate of the icing is defined and expressed by the following formula:

$$
R_{S}=\frac{S_{i}-S_{i-1}}{\Delta t},
$$

where $R_{s}$ is the growth rate of the icing, $S_{i}$ is the icing area at the icing time of $t_{i}, S_{i-1}$ is the icing area at the icing time of $t_{i-1}$, and $\Delta t$ is the time interval between $t_{i}$ and $t_{i-1}$.

5.2. Analysis of the Dimensionless Icing Area. To assess the difference in the icing states under static and rotating conditions, the experimental data and the laws of icing acquired from these two kinds of states are analyzed. The dimensionless icing area plotted against icing time length in the static state is shown in Figure 18.

As shown in Figure 18, the dimensionless icing area at a setting angle of $0^{\circ}$ is larger than the dimensionless icing area at a setting angle of $5^{\circ}$. Under these conditions, the dimensionless icing areas both increase with an increase in the length of the icing time. In addition, the slopes of these curves both decrease with an increase in the icing time because the thermal conductivity decreases with an increase in icing, layer by layer. In the initial stage, water droplets contact the blade surface directly and freeze into rime ice, because the aluminum has 


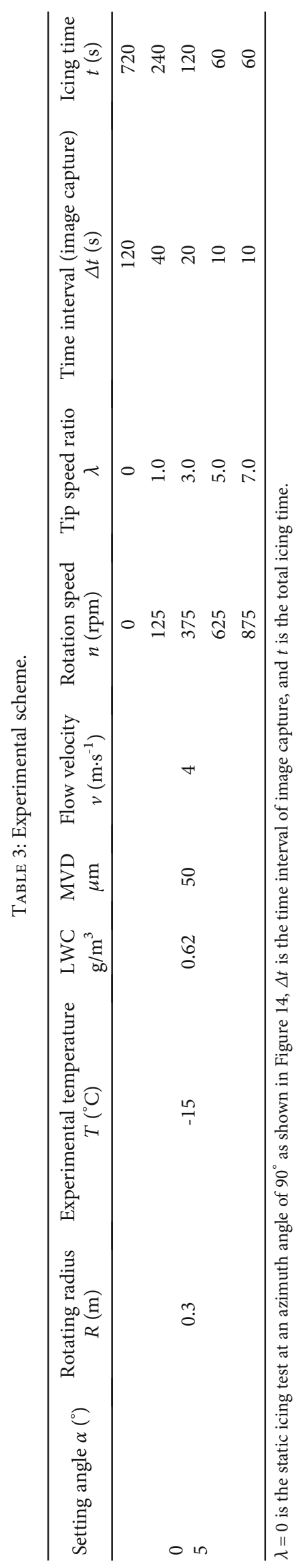



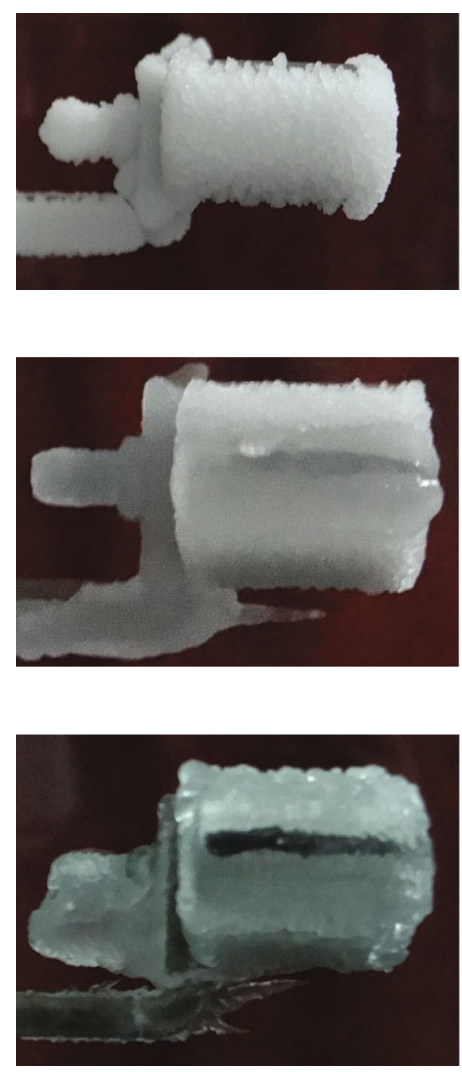

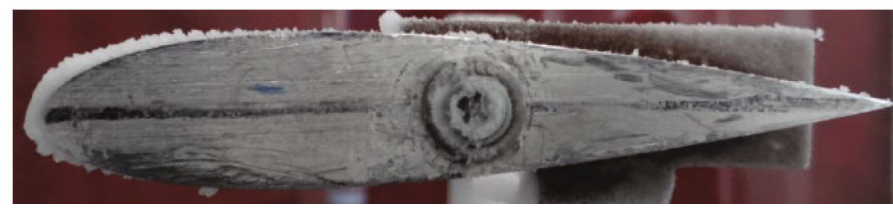

(a) Rime ice

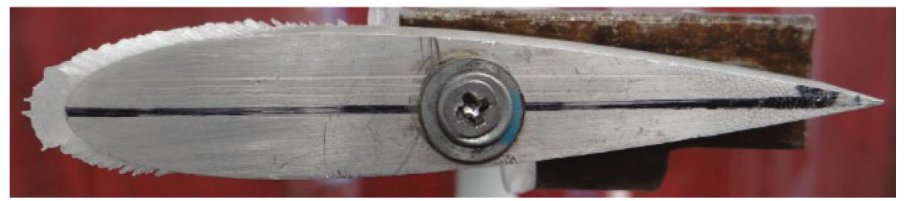

(b) Mixing ice

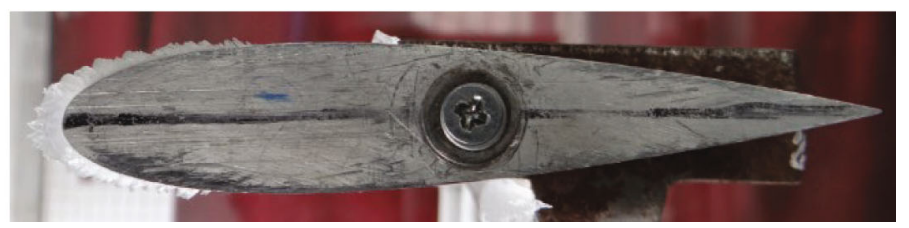

(c) Glaze ice

Figure 15: Types of ice.

a high thermal conductivity coefficient. The heat transfers from the water droplets to the blade in a short time. With an increase in the ice layer, the blade surface is fully covered by an ice layer with a low thermal conductivity coefficient, and the heat transfer from the water droplets to the ice layer requires more time. Therefore, the dimensionless icing area grows slowly with an increase in the icing test time.

The dimensionless icing areas plotted against icing time under different tip speed ratios are shown in Figure 19.

As shown in Figure 19, the dimensionless icing area increases with an increase in icing test time. The higher the rotational speed of the blade is, the larger the icing area is, because when the rotation speed increases, the blade can collect more water droplets per unit time. In addition, the dimensionless icing area at a setting angle of $5^{\circ}$ is higher than the dimensionless icing area at a setting angle of $0^{\circ}$ in the same length of icing time because the frontal area at a setting angle of $5^{\circ}$ is larger than the frontal area at a setting angle of $0^{\circ}$. Under this condition, more droplets can be captured by the blade in the same length of time. When the tip speed ratio is higher than 5, the growth rate of the dimensionless icing area varies slightly. The growth rate is in the range of $0.0013 \sim 0.0014$ when the setting angle is $0^{\circ}$. When the setting angle is $5^{\circ}$, the growth rate is in the range of $0.002 \sim 0.003$.

5.3. Analysis of the Growth Rate of Icing. The growth rates of icing plotted against icing test time in the static state are shown in Figure 20.
As shown in Figure 20, the change laws of growth rates of icing under different setting angles have two stages. They both increase first and then decrease. In the first stage, the growth rates of icing rise rapidly and reach the maximum value. The maximum growth rates are approximately 1.1 and 1.4 under setting angles of $0^{\circ}$ and $5^{\circ}$, respectively. In the second stage, the growth rates of icing decrease rapidly and finally tend to stabilize. In addition, the growth rate of icing at a setting angle of $5^{\circ}$ is higher than that at a setting angle of $0^{\circ}$ in the initial stage. With an increase in icing time, the growth rate of icing at a setting angle of $5^{\circ}$ decreases more rapidly than that at a setting angle of $0^{\circ}$ because the thermal conductivity decreases with an increase in the thickness of the ice layer. In the first stage, the water droplets directly contact the blade surface and freeze into ice in a short time because of the high thermal conductivity of the aluminum material. Therefore, the growth rates of icing increase rapidly at both setting angles. However, the blade at a setting angle of $5^{\circ}$ collects more water droplets than the blade at a setting angle of $0^{\circ}$ per unit time. The growth rate of icing at a setting angle of $5^{\circ}$ increases more quickly than that at a setting angle of $0^{\circ}$. In the second stage, because the ice layer on the blade surface at a setting angle of $5^{\circ}$ is thicker than the ice layer on the blade surface at a setting angle of $0^{\circ}$, the thermal conductivity of the blade is lower. Therefore, the growth rate of icing at this setting angle decreases more rapidly than the growth rate of icing at a setting angle of $0^{\circ}$. 


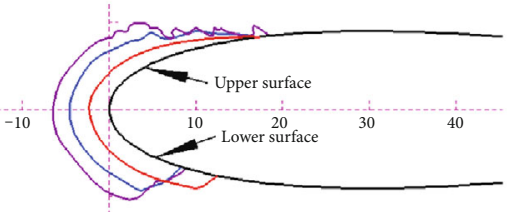

$$
\begin{aligned}
& \alpha=0^{\circ} \\
& n=0 \mathrm{rpm} \\
& -t=120 \mathrm{~s} \\
& -t=240 \mathrm{~s} \\
& -t=360 \mathrm{~s}
\end{aligned}
$$
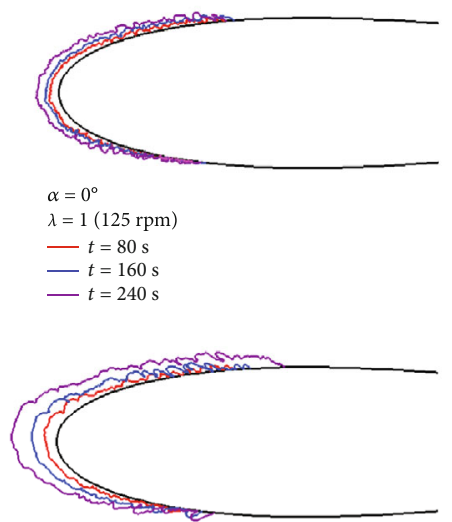

$\alpha=0^{\circ}$

$\lambda=3(375 \mathrm{rpm})$

$-t=40 \mathrm{~s}$

$-t=80 \mathrm{~s}$

$-t=120 \mathrm{~s}$

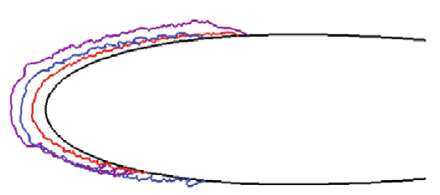

$\alpha=0^{\circ}$

$\lambda=5(625 \mathrm{rpm})$

$-t=20 \mathrm{~s}$

$-t=40 \mathrm{~s}$

$-t=60 \mathrm{~s}$

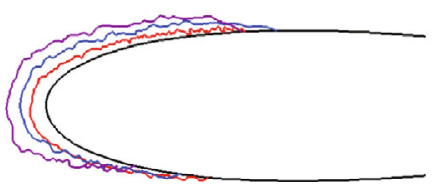

$\alpha=0^{\circ}$

$\lambda=7(875 \mathrm{rpm})$

$-t=20 \mathrm{~s}$

$\begin{aligned}-t & =40 \mathrm{~s} \\ -t & =60 \mathrm{~s}\end{aligned}$

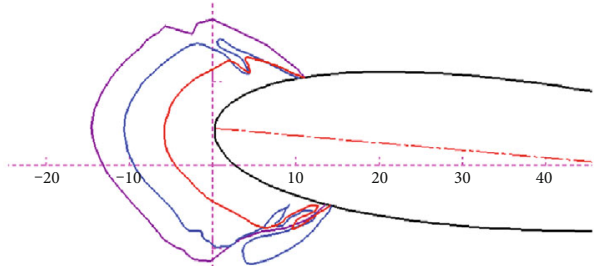

$\alpha=5^{\circ}$

$n=0 \mathrm{rpm}$

$-t=240 \mathrm{~s}$

$-t=480$

$\begin{aligned} t & =480 \\ -t & =720\end{aligned}$

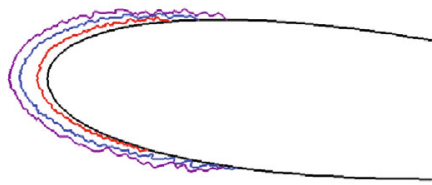

$\alpha=5^{\circ}$

$\lambda=1(125 \mathrm{rpm})$

- $t=80 \mathrm{~s}$

$t=160 \mathrm{~s}$
$-t=240 \mathrm{~s}$

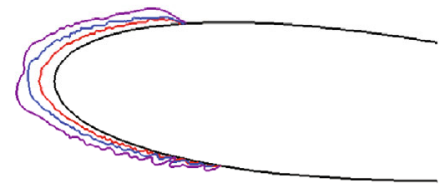

$\alpha=5^{\circ}$

$\lambda=3(375 \mathrm{rpm})$

$-t=40 \mathrm{~s}$

— $t=80 \mathrm{~s}$

- $t=120 \mathrm{~s}$

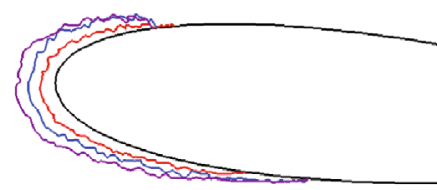

$\alpha=5^{\circ}$

$\lambda=5(625 \mathrm{rpm})$

$-t=20 \mathrm{~s}$

$\begin{aligned}-t & =40 \mathrm{~s} \\ -t & =60 \mathrm{~s}\end{aligned}$

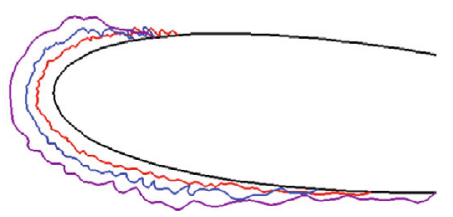

$\alpha=5^{\circ}$

$\lambda=7(875 \mathrm{rpm})$

- $t=20 \mathrm{~s}$

- $t=40 \mathrm{~s}$

Figure 16: Icing shapes and distributions under different conditions.

The growth rates of icing plotted against the icing test time under different tip speed ratios are shown in Figure 21.

As shown in Figure 21, the growth rates of icing are both low and fluctuating at two setting angles when the tip speed ratio $\lambda$ is 1 . In addition, the higher the tip speed ratio is, the higher the growth rate of icing is at each setting angle, because more droplets can be captured by the blade per unit time as the tip speed ratio increases. When the tip speed ratios are high (such as 5 and 7), the growth rates of icing increase rapidly in the initial stage and then decrease dramatically in a short time. The maximum growth rates of icing are both 2.8 because the thermal conductivity decreases with an increase in the thickness of the ice layer, which is similar to the change rule under static conditions. Moreover, the 


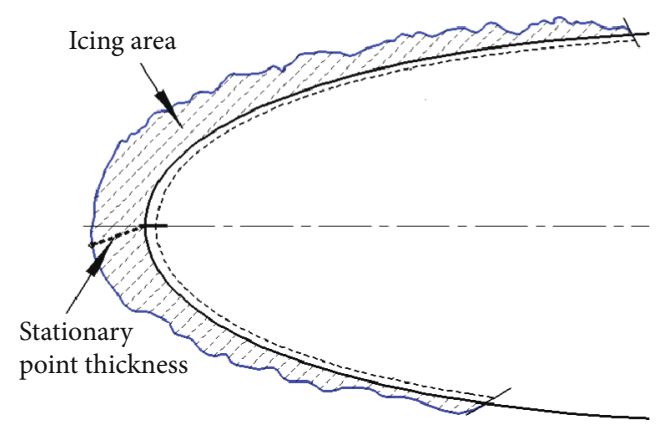

FIgUre 17: Typical characteristics of icing shape.

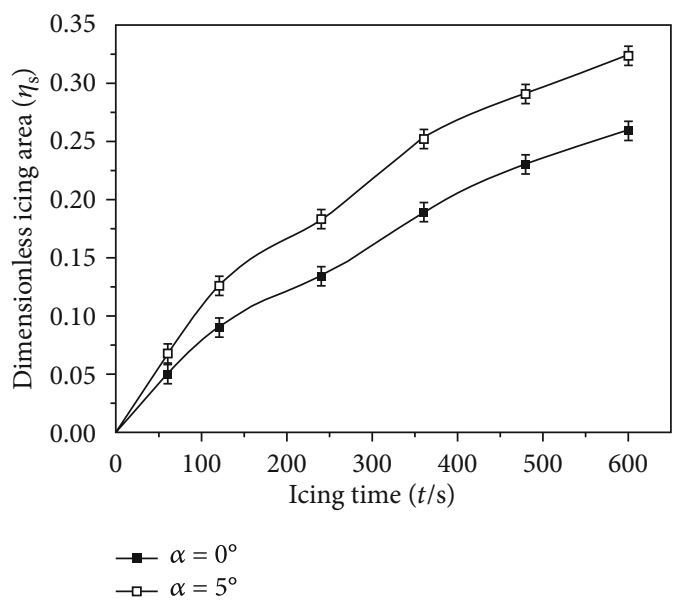

FIGURE 18: Dimensionless icing area in static states.

growth rate of icing at the tip speed ratio of 7 decreases more rapidly than that at a tip speed ratio of 5 because when the tip speed ratio is 7 , the water droplets contacting the blade surface are subject to a higher centrifugal force before freezing. Under these conditions, more water droplets converge and are thrown out from the blade surface.

5.4. Analysis of Stationary Point Thickness. The dimensionless stationary point thicknesses plotted against the icing test time at two setting angles in the static state are shown in Figure 22.

As shown in Figure 22, the dimensionless stationary point thicknesses increase with an increase in icing test time. The dimensionless stationary point thickness at a setting angle of $5^{\circ}$ is higher than that at a setting angle of $0^{\circ}$ in the same time length of the icing test. The slopes of these two curves decrease with an increase in the icing time because the blade has a larger frontal area at a setting angle of $5^{\circ}$, and more water droplets are collected by the frontal area. Therefore, the dimensionless stationary point thickness is higher under these conditions.

The dimensionless stationary point thicknesses plotted against the icing test time under different tip speed ratios are shown in Figure 23.

As shown in Figure 23, the dimensionless stationary point thicknesses increase with an increase in the icing test time under all tip speed ratios. The faster the rotation speed is, the higher the dimensionless stationary point thickness is. At the same tip speed ratio, the dimensionless stationary point thickness at a setting angle of $5^{\circ}$ is higher than that of the blade at a setting angle of $0^{\circ}$. In addition, when the tip speed ratios (such as 5 and 7) are higher, the dimensionless stationary point thicknesses are approximately the same. The key factors resulting in the above change law are the rotating speed of the blade and the difference in the thermal conductivity between the aluminum and the ice layer. With an increase in the tip speed ratio, the blade collects more water droplets per unit time, and the dimensionless stationary point thickness increases with rotational speed. As the tip speed ratios become higher, the water droplets contacting the blade surface cannot freeze in a short time because of the low thermal conductivity of the ice layer. Then, they are thrown out by centrifugal force, such that the growth rate of the dimensionless stationary point thickness increases slowly and is approximately the same under a high tip speed ratio. When the tip speed ratio is higher than 5 , the growth rate of the dimensionless stationary point thicknesses varies slightly. When the setting angle is $0^{\circ}$, the growth rate is in the range of 0.0007 0.0009. When the setting angle is $5^{\circ}$, the growth rate is in the range of $0.0007 \sim 0.0008$.

5.5. Uncertainty Analysis. As shown from Figures 18-23, there are errors in the dimensionless icing area, the growth rate of icing, and the dimensionless stationary point thicknesses. The errors in these parameters are calculated by formulae (7), (8), and (9).

$$
\Delta \eta_{s}=\frac{S_{\max }-S_{\min }}{S_{b}}
$$

where $\Delta \eta_{s}$ is the error of the dimensionless icing area, $S_{\max }$ is the maximum value of the cross-sectional area of the icing layer in each condition, and $S_{\min }$ is the minimum value of the cross-sectional area of the icing layer in each condition.

$$
\Delta \eta_{\sigma}=\frac{\sigma_{\max }-\sigma_{\min }}{c},
$$

where $\Delta \eta_{\sigma}$ is the error of dimensionless stationary point thicknesses, $\sigma_{\max }$ is the maximum value of stationary point thickness in each condition, and $\sigma_{\min }$ is the minimum value of stationary point thickness in each condition.

$$
\Delta R_{S}=\sqrt{\left(\Delta S_{i}\right)^{2}+\left(\Delta S_{i-1}\right)^{2}},
$$

where $\Delta R_{s}$ is the error of growth rate of icing, $\Delta S_{i}$ is the error of $S_{i}$ (the icing area at the icing time of $t_{i}$ ), and $\Delta S_{i-1}$ is the error of $S_{i}$ (the icing area at the icing time of $t_{i-1}$ ).

The factors that result in the experimental errors may be concluded as follows:

(1) The fluctuation of wind speed in the test section. The fluctuation of the rotating velocity of an electric fan 


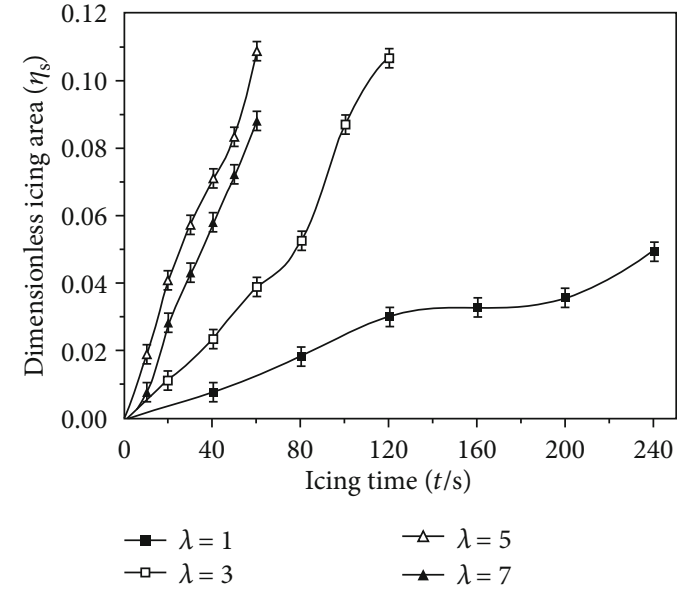

(a) $\alpha=0^{\circ}$

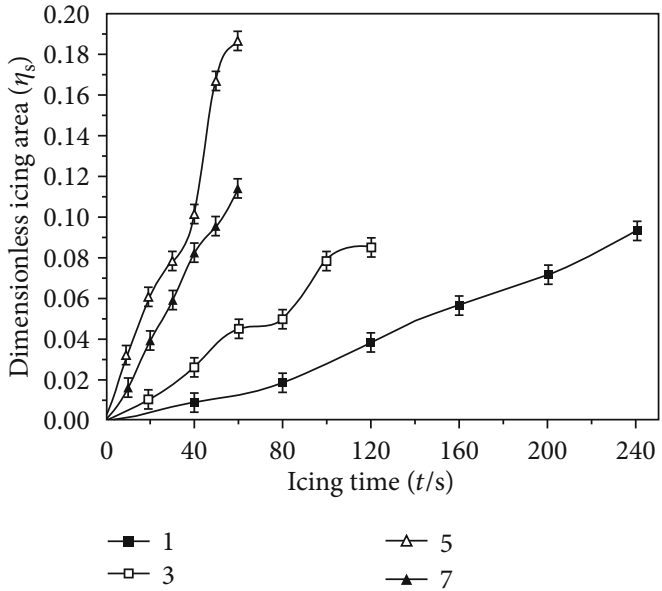

(b) $\alpha=5^{\circ}$

FIGURE 19: Dimensionless icing areas under rotating states.

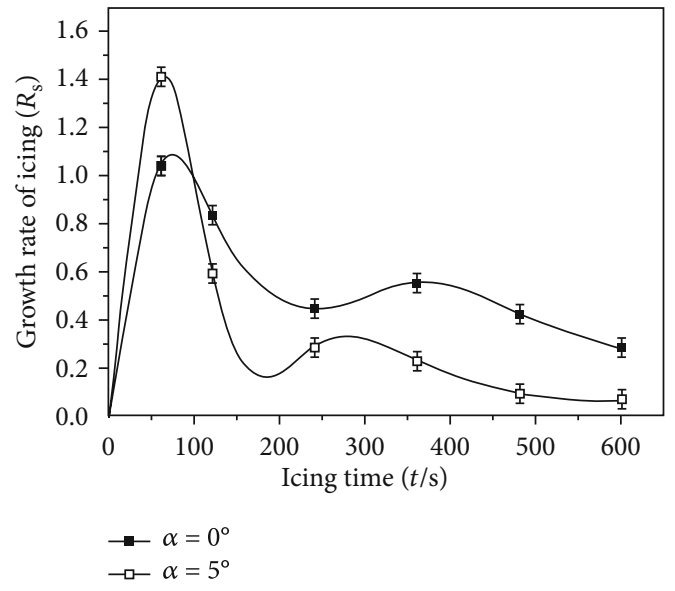

FIGURE 20: Growth rate of icing in static states.

and the blockage effect of the wall may result in the variation of wind speed. It slightly influences the icing shape of the airfoil blade

(2) The fluctuation of water pressure in the spraying system. When the water pump is working, the compression of water and the deformation of the pipe may result in a slight variation of pressure. It results in the changes of liquid water content (LWC) and medium volume diameter (MVD), which may lead to errors in the above parameters

(3) The errors in taking and processing the pictures. In this paper, the 2-D icing shapes were acquired by processing pictures, which were captured by a highspeed camera. In the procedure of acquiring icing shape, picture processing errors happened. For example, the end face of the icing blade might not be absolutely parallel to the lens. In addition, the drawing of icing shape by the spline curve in CAD also resulted in errors

\section{Discussions}

According to the above experimental results, the icing type changes from rime ice to glaze ice with an increase in the rotational speed. The growth rate of icing increases first and then decreases with an increase in the icing test time because of change in the thermal conductivity during the process of icing on the blade surface, which influences the characteristics of heat transfer. In the process of icing, there are three kinds of heat transfer models for the blade surface: heat convection between the ice layer and air, heat convection between the ice layer and the blade, and heat convection between the ice layers. In these models, the thermal conductivity coefficient of aluminum is $237 \mathrm{~W} /(\mathrm{m} \cdot \mathrm{K})$, and the thermal conductivity coefficients of air and ice are only $0.024 \mathrm{~W} /(\mathrm{m} \cdot \mathrm{K})$ and $2.22 \mathrm{~W} /(\mathrm{m} \cdot \mathrm{K})$, respectively. Therefore, the heat transfer model between the ice layer and the blade is the key heat transfer model.

Under the same icing conditions, the process of icing can be divided into two stages: the initial icing stage and the growing icing stage, as shown in Figure 24.

$\dot{E}_{\text {so }}$ is the energy transferred from the water layer to the blade during freezing, $\dot{H}_{\mathrm{va}}$ is the evaporation heat of the water layer or the ice layer into the air, $\dot{H}_{\mathrm{ou}}$ is the energy of the water droplets flowing out of the icing body, $\dot{H}_{\text {in }}$ is the energy of the water droplets flowing into the icing body, $\dot{H}_{\text {im }}$ is the impacting kinetic energy into the heat flux of the water layer, $\dot{Q}_{f}$ is the friction heating energy between the air and the water layer, $\dot{Q}_{c}$ is the heat of convective flow between the icing body and the environment, and $\dot{Q}_{k}$ is the heat transfer between the ice and water.

At the initial stage of icing, the heat energy in the water droplets conducts into the blade in a short time because of the high thermal conductivity of the aluminum. The ice layer grows layer by layer with an increase in the icing time. Under these conditions, the heat energy in the water droplets conducts into the blade via the ice layer. 


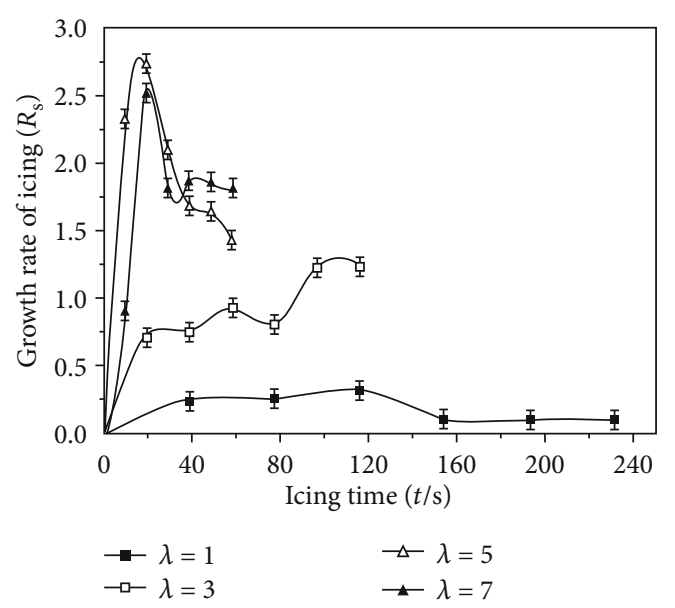

(a) $\alpha=0^{\circ}$

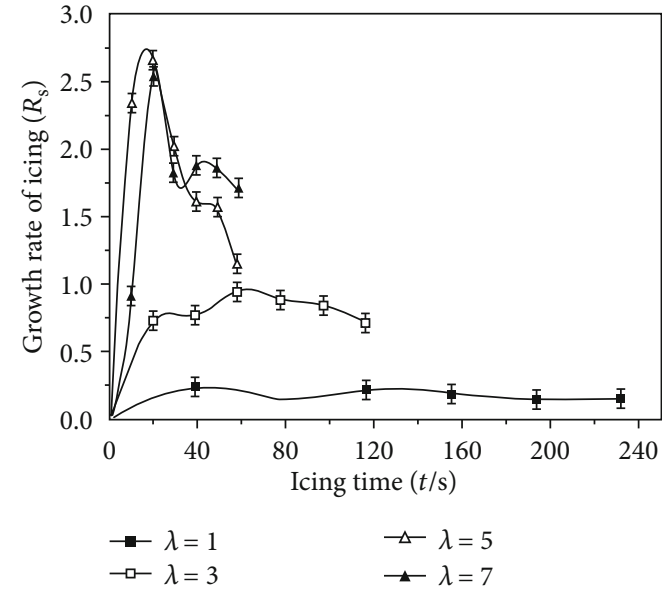

(b) $\alpha=5^{\circ}$

FIGURE 21: Growth rate of icing under different tip speed ratios.

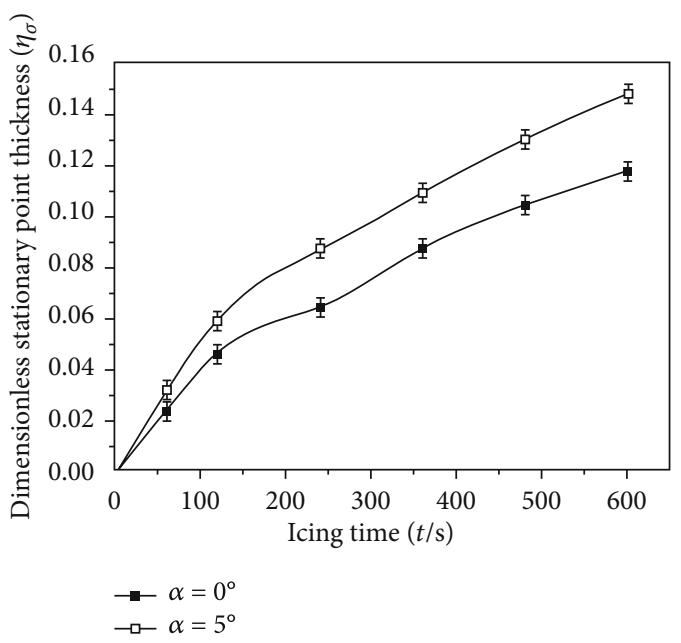

FIGURE 22: Dimensionless stationary point thicknesses in static states.

The growth rate of the icing and other characteristic parameters are greatly influenced by the change in the heat transfer model.

The heat transfer model is influenced by the tip speed ratio. When the tip speed ratios are low and static, the heat energy in the supercooled water droplets can be conducted into the blade body completely. With an increase in the tip speed ratio, more supercooled water droplets are collected by the blade per unit time. In theory, more heat energy should be conducted into the blade body. However, the thermal conductivity coefficients of aluminum and ice are constant. The icing blade has a superior limit of thermal conductivity. Therefore, the icing blade cannot absorb all the heat energy in the water droplets collected by the blade under a high tip speed ratio. Then, a supercooled water layer occurs on the blade surface. The water layer flows under the effect of centrifugal force, and the icicles occur under high tip speed ratios such as five and seven.

\section{Conclusions}

In this paper, the experiment on icing on the blade with NACA0018 airfoil was carried out in a self-developed and constructed icing wind tunnel, and four conclusions can be summarized as follows:

(1) An icing wind tunnel experimental system was set up based on a conventional wind tunnel, and the conditional parameters, such as temperature, flow velocity, LWC, and MVD, were calibrated. The experimental temperature in the test section was $-15^{\circ} \mathrm{C}$. The uniform region of LWC represents approximately $75 \%$ of the total cross-section in the test section. The MVD in the test section is approximately $50 \mu \mathrm{m}$

(2) The tip speed ratio and the setting angle influence the type of icing on the blade surface. Rime ice generates under a low tip speed ratio, and mixing ice and glaze ice are present under the condition of a high tip speed ratio. When the setting angle is $0^{\circ}$, rime ice generates at tip speed ratios of 0 and 1 , and mixing ice is present at tip speed ratios of 3,5 , and 7 . When the setting angle is $5^{\circ}$, rime ice also generates at low tip speed ratios such as 0 and 1 . Mixing ice is present at tip speed ratios of 3 and 5, and glaze ice occurs at the tip speed ratio of 7

(3) The setting angle influences the icing area on the blade surface. When the setting angle is $0^{\circ}$, the areas covered by the ice layer on the upper blade surface are larger than the areas covered by the ice layer on the lower surface. The area covered by the ice layer on the upper blade surface accounts for approximately $30 \%$ of the total upper blade surface area, and the area covered by the ice layer on the lower blade surface is approximately $15 \%$ of the total lower blade surface area. When 


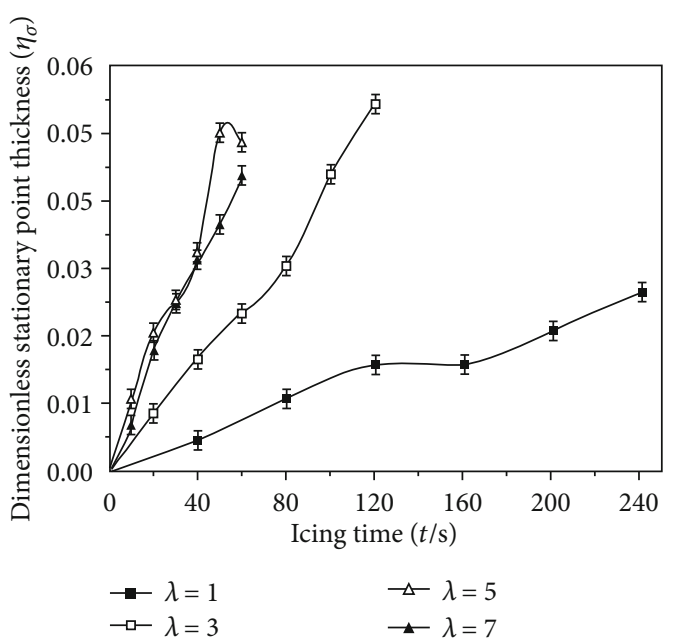

(a) $\alpha=0^{\circ}$

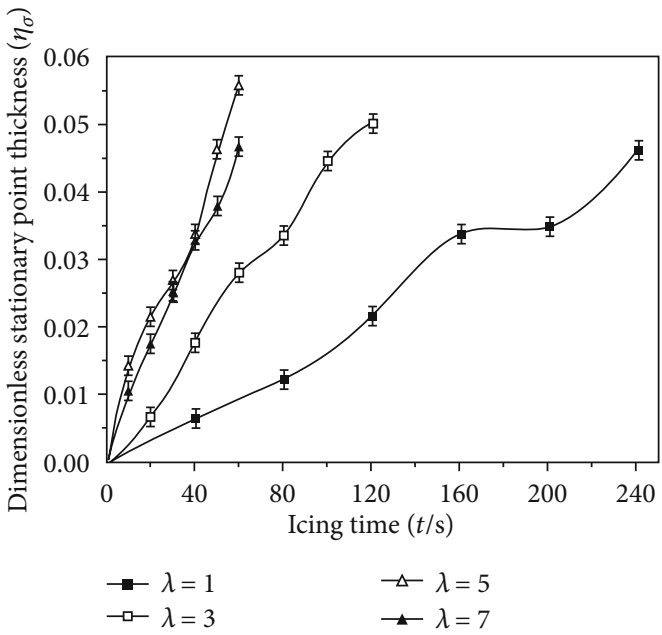

(b) $\alpha=5^{\circ}$

Figure 23: Dimensionless stationary point thicknesses under different tip speed ratios.

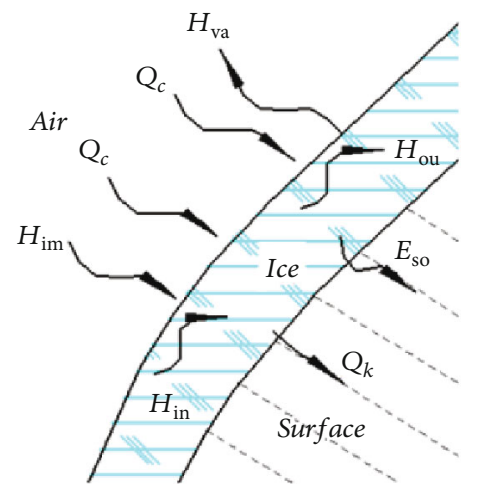

(a) Heat transfer model in the initial icing stage

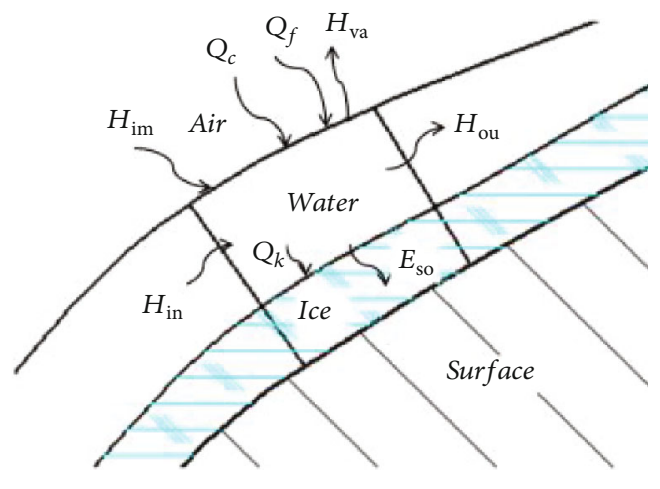

(b) Heat transfer model in the growing icing stage

Figure 24: Heat transfer model in different icing stages.

the setting angle is $5^{\circ}$, the area covered by the ice layer on the upper blade surface is smaller than the area covered by the ice layer on the lower surface

(4) The dimensionless icing area and the dimensionless stationary point thickness, under static conditions and rotating conditions, increase with an increase in icing time. The growth rates of icing under different tip speed ratios and setting angles increase first and then decrease until stability is reached. The higher the tip speed ratio is, the larger the icing area and stationary point thickness are. The icing area and stationary point thickness at a setting angle of $5^{\circ}$ are both larger than those at a setting angle of $0^{\circ}$. When the tip speed ratio is higher than 5 , the growth rate of the dimensionless icing area varies slightly. The growth rates are in the ranges of $0.0013 \sim 0.0014$ and $0.002 \sim 0.003$ at setting angles of $0^{\circ}$ and $5^{\circ}$, respectively. Similarly, the growth rates of the dimensionless stationary point thicknesses are in the ranges of 0.0007 0.0009 and 0.0007 0.0008 at setting angles of $0^{\circ}$ and $5^{\circ}$, respectively

\section{Nomenclature}

C: $\quad$ Chord length of blade

$E_{\mathrm{so}}$ : Energy transferred from the water layer to the blade during freezing

$H: \quad$ Thickness of blade

$\dot{H}_{\mathrm{im}}$ : Impacting kinetic energy into the heat flux of the water layer

$\dot{H}_{\text {in }}$ : Energy of the water droplets flowing into the icing body

$\dot{H}_{\mathrm{ou}}$ : Energy of the water droplets flowing out of the icing body

$\dot{H}_{\mathrm{va}}$ : Evaporation heat of the water layer or the ice layer into the air

$h$ : $\quad$ Thickness of icing layer

LWC: Liquid water content

MVD: Medium volume diameter

$n_{i}$ : $\quad$ Mass ratio of water droplets

$\dot{Q}_{c}: \quad$ Heat of convective flow between the icing body and the environment

$\dot{Q}_{f}: \quad$ Friction heating energy between the air and the water layer

Heat transfer between the ice and water 
$\dot{Q_{k}}$ :

$R_{s}: \quad$ Growth rate of the icing

$\Delta R_{s}: \quad$ Error of growth rate of icing

$S: \quad$ Cross-sectional area of the icing layer

$S_{b}$ : Cross-sectional area of the blade

$S_{i}: \quad$ Icing area at the icing time of $t_{i}$

$S_{i-1}: \quad$ Icing area at the icing time of $t_{i-1}$

$\Delta S_{i}$ : $\quad$ Error of $S_{i}$ (the icing area at the icing time of $t_{i}$ )

$\Delta S_{i-1}$ : Error of $S_{i}$ (the icing area at the icing time of $t_{i-1}$ )

$S_{\max }$ : Maximum value of the cross-sectional area of the icing layer in each condition

$S_{\min }$ : Minimum value of the cross-sectional area of the icing layer in each condition

$T: \quad$ Experimental temperature in the test section

$t$ : Total icing time

$\Delta t: \quad$ Time interval of image capture

$U: \quad$ Tangential velocity of the blade

$v$ : $\quad$ Flow velocity in the test section

$\alpha$ : $\quad$ Setting angle of blade

$\beta$ : $\quad$ Coefficient of local water droplet collection in the calibration experiment

$\beta_{0}$ : Coefficient of local water droplet collection in the simulation

$\eta: \quad$ Dynamic stability of wind speed in wind tunnel

$\eta_{s}: \quad$ Dimensionless icing area

$\eta_{\sigma}: \quad$ Dimensionless stationary point thicknesses

$\Delta \eta_{s}: \quad$ Error of dimensionless icing area

$\Delta \eta_{\sigma}: \quad$ Error of dimensionless stationary point thicknesses

$\lambda: \quad$ Tip speed ratio of rotating blade

$\mu_{I}: \quad$ Pressure field coefficient of wind tunnel

$\sigma: \quad$ Stationary point thickness

$\sigma_{\max }:$ Maximum value of stationary point thickness in each condition

$\sigma_{\min }:$ Minimum value of stationary point thickness in each condition.

\section{Data Availability}

The data used to support the findings of this study are available from the corresponding author upon request.

\section{Conflicts of Interest}

The authors declare that they have no conflicts of interest.

\section{Acknowledgments}

This research was supported by the National Natural Science Foundation of China (NSFC, No. 51976029 and 51576037) and the Key Laboratory of Aircraft Icing and the Anti/Deicing Open Subject Foundation of China (AIADL20180303).

\section{References}

[1] Y. Zhang, N. Tang, Y. Niu, and X. Du, "Wind energy rejection in China: current status, reasons and perspectives," Renewable and Sustainable Energy Reviews, vol. 66, pp. 322-344, 2016.

[2] Y. Li, S. Zhao, K. Tagawa, and F. Feng, "Starting performance effect of a truncated-cone-shaped wind gathering device on small-scale straight-bladed vertical axis wind turbine," Energy Conversion and Management, vol. 167, pp. 70-80, 2018.

[3] L. Ville, "Available technologies of wind energy in cold climates," IEA Wind Task, vol. 19, 2016.

[4] L. Shu, J. Liang, Q. Hu, X. Jiang, X. Ren, and G. Qiu, "Study on small wind turbine icing and its performance," Cold Regions Science and Technology, vol. 134, pp. 11-19, 2017.

[5] I. Paraschivoiu, Aircraft Icing, John Wiley and Sons, Inc, 2018.

[6] Y. Cao, W. Tan, and Z. Wu, "Aircraft icing: an ongoing threat to aviation safety," Aerospace Science and Technology, vol. 75, pp. 353-385, 2018.

[7] Y. Li, K. Tagawa, F. Feng, Q. Li, and Q. He, "A wind tunnel experimental study of icing on wind turbine blade airfoil," Energy Conversion and Management, vol. 85, pp. 591-595, 2014.

[8] Y. Xian, Numerical computation of aircraft icing and study on icing test scaling law, China Aerodynamics Research and Development Center Graduate School, Mianyang, China, 2007.

[9] G. L. Taylor, Notes on Possible Equipment and Technique for Experiments on Icing on Aircraft, Cambridge at the Unuversity Press, 1940.

[10] L. Chen, Y. Zhang, and Q. Wu, "Heat transfer optimization and experimental validation of anti-icing component for helicopter rotor," Applied Thermal Engineering, vol. 127, pp. 662670, 2017.

[11] G. A. Ruff, Analysis and Verification of the Icing Scaling Equations, Arnold Engineering Development Center, 1986.

[12] S. L. Wang, Numerical simulation and icing wind tunnel test study on icing distribution on rotating blade of horizontal axis wind turbine, Northeast Agricultural University, 2017.

[13] R. Flemming, P. Alldridge, and R. Doeppner, "Artificial icing tests of the S-92A helicopter in the McKinley Climatic Laboratory," in 42nd AIAA Aerospace Sciences Meeting and Exhibit, Reno, Nevada, January 2013.

[14] R. M. Waldman and H. Hu, "High-speed imaging to quantify transient ice accretion process over an airfoil," Journal of Aircraft, vol. 53, no. 2, pp. 1-9, 2016.

[15] Y. Liu, L. Li, W. Chen, W. Tian, and H. Hu, “An experimental study on the aerodynamic performance degradation of a UAS propeller model induced by ice accretion process," Experimental Thermal and Fluid Science, vol. 102, pp. 101-112, 2019.

[16] Y. Han, J. Palacios, and S. Schmitz, "Scaled ice accretion experiments on a rotating wind turbine blade," Journal of Wind Engineering and Industrial Aerodynamics, vol. 109, pp. 5567, 2012.

[17] A. G. Kraj and E. L. Bibeau, "Phases of icing on wind turbine blades characterized by ice accumulation," Renewable Energy, vol. 35, no. 5, pp. 966-972, 2010.

[18] Y. Li, S. Wang, Q. Liu, F. Feng, and K. Tagawa, "Characteristics of ice accretions on blade of the straight-bladed vertical axis wind turbine rotating at low tip speed ratio," Cold Regions Science and Technology, vol. 145, no. 13, pp. 1-13, 2018.

[19] Y. Li, C. Sun, Y. Jiang, and F. Feng, "Scaling method of the rotating blade of a wind turbine for a rime ice wind tunnel test," Energies, vol. 12, no. 4, p. 627, 2019.

[20] Y. Li, S. Wang, Y. Zheng, Q. D. Liu, F. Feng, and K. Tagawa, "Design of wind tunnel experiment system for wind turbine icing by using natural low temperature," Journal of experiments in Fluid Mechanics, vol. 30, no. 2, pp. 54-58, 2016. 
[21] X. Yi, Y. W. Gui, Y. X. Du, G. L. Zhu, and J. Li, "Study on the method of droplet diameter calibration in icing wind tunnel," Journal of Experiments in Fluid Mechanics, vol. 24, no. 5, pp. 36-46, 2010.

[22] H.-P. Chang and K. R. Kimble, "Influence of multidroplet size distribution on icing collection efficiency," in 21st Aerospace Sciences Meeting, Reno, NV, USA, January 1983. 